\title{
Comparison of Effect on Sicca Symptoms of Anticentromere Antibody-positive Sjögren Syndrome and Primary Sjögren Syndrome Alone
}

\author{
Yifan Li(D) and Arthur A.M. Bookman
}

\begin{abstract}
Objective. To determine whether positive anticentromere antibody (ACA) serology affects the severity of sicca symptoms in patients with primary Sjögren syndrome (pSS).

Methods. Evaluation to detect subjective and objective sicca symptoms included questionnaires, physical examination, and pathology. Cases of pSS were classified according to the 2002 American-European Consensus Group (AECG) criteria. All patients were evaluated for presence of anti-Ro, anti-La, and ACA serology. Patients with pSS were categorized into ACA+ SS and ACA-SS. The groups were compared for measures of severity of oral and ocular sicca.

Results. The pSS group had 446 patients, of whom 26 were ACA+ SS. Subjective ocular sicca measured $7.0 \pm 2.4$ (out of 10) in ACA+ SS and $6.4 \pm 2.6$ in ACA- SS $(\mathrm{p}=0.197)$. Objective ocular sicca measured $3.2 \mathrm{~mm} \pm 1.8 \mathrm{~mm} / 5 \mathrm{~min}$ in ACA+SS and $4.2 \mathrm{~mm} \pm 4.4 \mathrm{~mm} / 5 \mathrm{~min}$ in ACA- SS (p = 0.038). Subjective oral sicca measured 8.5 \pm 1.4 in ACA+ SS and 6.7 \pm 2.4 in ACA-SS ( $<<0.001)$. Objective oral sicca measured $0.1 \mathrm{ml} \pm 0.2 \mathrm{ml} / 15 \mathrm{~min}$ in ACA+SS and $0.4 \mathrm{ml} \pm 1.0 \mathrm{ml} / 15 \mathrm{~min}$ in ACA-SS $(\mathrm{p}<0.001)$. Only $35 \%$ of ACA + patients with SS were anti-Ro-positive or anti-La-positive compared with $77 \%$ of the ACA- patients with SS ( $<<0.001)$. There was no significant difference in minor salivary gland fibrosis or focus scores between ACA+ SS and ACA- patients with SS.

Conclusion. ACA+ SS is associated with more severe objective ocular sicca and more severe subjective and objective oral sicca compared to ACA-SS. The majority of ACA+ patients with SS meet AECG criteria for pSS despite negative serology for anti-Ro/La antibodies. (First Release March 1 2020; J Rheumatol 2020;47:876-80; doi:10.3899/jrheum.190462)
\end{abstract}

Key Indexing Terms:

SJÖGREN SYNDROME

SICCA SYMPTOMS

\section{ANTICENTROMERE ANTIBODY PRIMARY SJÖGREN SYNDROME}

The purpose of our study was to determine whether the presence of anticentromere antibodies (ACA) affects the severity of sicca signs and symptoms in patients diagnosed with primary Sjögren syndrome (pSS).

Sjögren syndrome (SS) is a chronic autoimmune disease that mainly affects the exocrine glands ${ }^{1}$. Sicca symptoms of xerophthalmia (ocular dryness) and xerostomia (oral dryness) are the most prevalent symptoms in $\mathrm{SS}^{1}$. In overlapping autoimmune disorders, there can be a clinical difference in the severity of at least one of the diseases ${ }^{2}$.

The American-European Consensus Group (AECG) classification criteria for SS defined secondary SS as the presence of signs and symptoms of dry eyes or dry mouth in

\footnotetext{
From the Multidisciplinary Sjögren's Clinic, University Health Network, University of Toronto, Toronto, Ontario, Canada.

Y. Li, XX, Multidisciplinary Sjögren's Clinic, University Health Network, University of Toronto; A.A. Bookman, XX, Multidisciplinary Sjögren's Clinic, University Health Network, University of Toronto.

This project was made possible with the support of the Sjögren's Society of Canada Summer Student Research Grant.

Address correspondence to Dr. A.A. Bookman, Toronto Western Hospital, University Health Network, 399 Bathurst St., Room 1E424, Toronto, Ontario M5T2S8, Canada.E-mail:dr.arthur.bookman@uhn.ca Accepted for publication September 27, 2019.
}

another well-defined major connective tissue disease ${ }^{3}$. In 2012, the American College of Rheumatology proposed new classification criteria for SS that challenged the distinction between primary and secondary $\mathrm{SS}^{4}$. The terms primary and secondary regarding SS have been debated because overlap in connective tissue disease is common. It can often be unclear which disease occurred first, or if one was secondary to the other ${ }^{5,6,7}$. This is as true of SS as it is with any other connective tissue disease. The presence of ACA has been reported in 1.4-10.85\% of patients with $\mathrm{pSS}^{7,8,9,10}$. Many of these patients have overlap features of limited cutaneous systemic sclerosis (lcSSc).

\section{MATERIALS AND METHODS}

Patients who were found at prescreening to have objective signs of dry eyes or dry mouth, positive anti-Ro or anti-La antibodies, or a history of parotitis were referred to the Multidisciplinary Sjögren's Clinic at University Health Network, Toronto, Canada, for further evaluation. All patients were evaluated according to a standardized protocol between 1992 and 2014. Evaluation included patients' global assessment of xerophthalmia and xerostomia on a standardized $10-\mathrm{cm}$ visual analog scale (VAS).

Demographic features were collected for each patient in the study population. Data were collected on subjective and objective xerophthalmia and xerostomia in patients diagnosed with SS.

Xerophthalmia was objectively measured using the Schirmer-I test 
$(\mathrm{S} 1 \mathrm{~T})^{11}$ and a van Bijsterveld scale for ocular staining using rose bengal or lissamine green ${ }^{12,13}$.

Unstimulated whole salivary flow (USSF) ${ }^{14}$ was measured after stopping all anticholinergic or sympathomimetic medication for $24 \mathrm{~h}$. All patients evaluated had a minor salivary gland (MSG) biopsy and the results were assessed by the same pathologist according to a protocol for the presence of focal lymphocytic sialadenitis, and a focus score was assigned. Degree of fibrosis was also graded. A score of 0 meant no fibrosis. A score of 3 meant confluent fibrosis. Extractable nuclear antigens (ENA) and ACA were assayed using the BioPlex Multiplex 2200 test kit, which relies on indirect immunofluorescence.

For our purposes we counted each of the CREST manifestations [calcinosis, Raynaud phenomenon (RP), esophageal dysmotility, sclerodactyly, and telangiectasia] as a "feature" of lcSSc. All patients were evaluated for CREST manifestations by history and physical examination. Radiographs of the hands were performed in all patients. The protocols of our study adhered to the Declaration of Helsinki.

We used the AECG criteria to classify $\mathrm{pSS}^{3}$. Patients who met the pSS classification for primary SS were further categorized into ACA+ SS and ACA-SS. Groups were compared to determine differences in the prevalence of sicca symptoms, severity of sicca symptoms, and serological markers including IgG quantification and anti-Ro and anti-La antibodies.

A 2-tailed Student $t$ test with heterogeneous variance was used to evaluate statistical significance when comparing differences between groups. A p value $<0.05$ was considered statistically significant. All statistical analyses were conducted using SAS 9.3.

Ethics approval was received from the University Health Network Research Ethics Board at the University of Toronto for our study with submission number 19-5454.0. Patients' written consent was waived because patient data was anonymized and no personal details could be identified.

\section{RESULTS}

Of 609 patients evaluated at the Multidisciplinary Sjögren's Clinic, 446 met the AECG classification criteria for pSS. Within the pSS group, there were 26 patients with positive ACA serology. These 26 patients (5.8\% of pSS group) were designated as ACA+ SS. There were 420 patients without ACA ( $94.2 \%$ of pSS group) and these were designated as ACA-SS.

The demographic characteristics of the 2 patient groups (ACA+ SS, ACA-SS) are summarized in Table 1. The process for patient enrollment is illustrated in Figure 1.

An additional 8 patients with ACA positivity were assessed in the Multidisciplinary Sjögren's Clinic because of complaints of dry eyes or mouth. These patients did not satisfy the AECG classification criteria for pSS. There was no statistically significant difference in the prevalence or severity of sicca symptoms among ACA+ patients regardless of whether criteria for pSS were met.

Among the 446 patients with pSS, on a VAS, the mean severity of ocular sicca symptoms was $7.0 \pm 2.4 / 10$ in patients with ACA+ SS and 6.4 $\pm 2.6 / 10$ in patients with ACA- SS $(\mathrm{p}=0.197$; Table 1). The mean S1T in patients with ACA+ SS was $3.2 \mathrm{~mm} \pm 1.8 \mathrm{~mm} / 5 \mathrm{~min}$ and in patients with ACA$\mathrm{SS}, 4.2 \mathrm{~mm} \pm 4.4 \mathrm{~mm} / 5 \mathrm{~min}(\mathrm{p}<0.05)$. However, there was no difference in the mean van Bijsterveld score between $\mathrm{ACA}+\mathrm{SS}(5.7 \pm 2.2 / 9)$ and ACA- SS $(5.6 \pm 2.3 / 9$; $\mathrm{p}=0.232)$.

On a VAS, the mean score for severity of oral sicca symptoms was $8.5 \pm 1.4 / 10$ in patients with ACA+ SS and $6.7 \pm 2.4 / 10$ in patients with ACA- SS ( $<<0.001$; Table 1). The mean value for USSF was $0.1 \mathrm{ml} \pm 0.2 \mathrm{ml} / 15 \mathrm{~min}$ for ACA+ SS and $0.4 \mathrm{ml} \pm 1.0 \mathrm{ml} / 15 \mathrm{~min}$ for ACA-SS. While this difference is statistically significant $(\mathrm{p}<0.001)$, there is an overlap in SD between the 2 groups. Similarly, a greater number of patients with ACA+ SS had a focus score $>1$ (92\%) compared with ACA- patients with SS (84\%). This difference was also highly significant ( $\mathrm{p}<0.001$; Table 1$)$. However, there was no statistically significant difference in the mean focus score between the ACA+ SS (focus score 5.5) and ACA-SS (focus score 4.0) groups. There was no significant difference in either the mean fibrosis score or the duration of xerophthalmia or xerostomia between the ACA+ SS and ACA-SS groups.

The CREST features in patients with ACA+ SS were not very severe or prevalent. Among patients with ACA+ SS, $58 \%$ had 2 or fewer stigmata of CREST syndrome, with RP and sclerodactyly being the predominant findings. Clinically, as expected, RP was more prevalent among patients with ACA+ SS (88\%) than patients with ACA- SS (28\%). This difference was highly significant $(\mathrm{p}<0.001)$. There were no statistically significant differences between the 2 groups regarding extraglandular features such as dental problems (decayed, missing, or filled teeth), parotitis, parotid gland swelling, lymphoma, vasculitis, or hypothyroidism.

Serological differences. Elevated IgG was seen in $24 \%$ of the patients with ACA+ SS and $57 \%$ of the patients with ACASS $(\mathrm{p}<0.001)$. There was a significant difference in the level of IgG between the ACA+ SS (12.4 g/l) and ACA-SS (19.4 $\mathrm{g} / \mathrm{l})$ groups $(\mathrm{p}<0.001)$. Only $35 \%$ of the patients with ACA+ SS had positive serology for anti-Ro or anti-La antibodies compared with $77 \%$ of the patients with ACA- SS $(\mathrm{p}<0.001)$. Nonetheless, these ACA-positive patients met the AECG classification criteria for $\mathrm{pSS}$, mainly based on an abnormal MSG biopsy.

\section{DISCUSSION}

The presence of overlapping autoimmune diseases is not uncommon in patients who are being evaluated for SS. There is no doubt that 2 overlapping autoimmune diseases influence one another. SSc (defined by clinical features such as sclerodactyly) overlapping with pSS (SSc-SS) was characterized by milder SSc, with a lower severity and prevalence of sclerodactyly, lung fibrosis, and systemic involvement ${ }^{7}$. In a French cohort, Salliot and colleagues found that among the SSc-SS group $(n=20)$, there was a greater frequency of RP, objective xerophthalmia, peripheral neuropathy, arthritis, and additional autoimmune disorders, especially primary biliary cholangitis, compared to the SS group ${ }^{7}$. Similar to the ACA+ SS group in our study, they found that their patients with SSc-SS had a statistically significantly lower prevalence of specific autoantibodies including rheumatoid factor, anti-Ro, and anti-La compared to patients with $\mathrm{SS}^{7}$. In their cohort,

$$
\text { Personal non-commercial use only. The Journal of Rheumatology Copyright @ } 2020 \text {. All rights reserved. }
$$


Table 1. Comparison between ACA+SS and ACA-SS.

\begin{tabular}{|c|c|c|c|}
\hline & $\mathrm{ACA}+\mathrm{SS}, \mathrm{n}=26$ & $\mathrm{ACA}-\mathrm{SS}, \mathrm{n}=420$ & $\mathrm{p}$ \\
\hline \multicolumn{4}{|l|}{ Demographics } \\
\hline Age, yrs, mean \pm SD & $55.7 \pm 10.5$ & $53.2 \pm 13.4$ & NS \\
\hline \multicolumn{4}{|l|}{ Sex } \\
\hline Male & 0 & 39 & NS \\
\hline Female & 26 & 381 & NS \\
\hline \multicolumn{4}{|l|}{ Clinical differences } \\
\hline Prevalence of RP $(\%)$ & 88 & 28 & $<0.001$ \\
\hline \multicolumn{4}{|l|}{ Serological markers } \\
\hline Prevalence of positive Ro/La antibodies (\%) & 35 & 77 & $<0.001$ \\
\hline Prevalence of elevated serum $\operatorname{IgG}(\%)$ & 24 & 57 & $<0.001$ \\
\hline Average levels of serum $\mathrm{IgG}, \mathrm{gm} / 1$ & 12.4 & 19.4 & $<0.001$ \\
\hline \multicolumn{4}{|l|}{ Xerophthalmia } \\
\hline Prevalence of xerophthalmia (\%) & 96 & 96 & NS \\
\hline Severity of xerophthalmia (on VAS, $\max 10$ ) & $7 \pm 2.4$ & $6.4 \pm 2.6$ & NS \\
\hline Average van Bijsterveld score & $5.7 \pm 2.2$ & $5.6 \pm 2.3$ & NS \\
\hline Schirmer-I test, $\mathrm{mm} / 5 \mathrm{~min}$ & $3.2 \pm 1.8$ & $4.2 \pm 4.4$ & $<0.05$ \\
\hline Duration, yrs, range & $5.4(0-20)$ & $7.5(0-50)$ & NS \\
\hline \multicolumn{4}{|l|}{ Xerostomia } \\
\hline Prevalence of xerostomia $(\%)$ & 100 & 98 & NS \\
\hline Severity of xerostomia (on VAS, $\max 10$ ) & $8.5 \pm 1.4$ & $6.7 \pm 2.4$ & $<0.001$ \\
\hline USSF, $\mathrm{ml} / 15 \mathrm{~min}$ & 0.1 & 0.4 & $<0.001$ \\
\hline Duration, yrs, range & $5.8(0-22)$ & $6.8(0-45)$ & NS \\
\hline \multicolumn{4}{|l|}{ Salivary gland biopsy } \\
\hline Focus score $\geq 1(\%)$ & 92 & 84 & $<0.001$ \\
\hline Average focus score & $5.5 \pm 4.3$ & $4.0 \pm 3.3$ & NS \\
\hline Average fibrosis score (out of 3 ) & $1.0 \pm 0.82$ & $1.1 \pm 0.68$ & NS \\
\hline
\end{tabular}

Normal serum IgG levels are 7-16 g/l in healthy patients. Rose bengal test evaluates ocular surface epithelial damage (out of 9). Schirmer-I test measures lacrimal gland production. Normal USSF $\geq 1.5 \mathrm{~mm} / 15 \mathrm{~min}$. Salivary gland biopsy positive result: $\geq 1$ focus per $4 \mathrm{~mm}^{2}$. SS: Sjögren syndrome; ACA: anticentromere antibodies; NS: not significant; RP: Raynaud phenomenon; VAS: visual analog scale; USSF: unstimulated whole salivary flow.

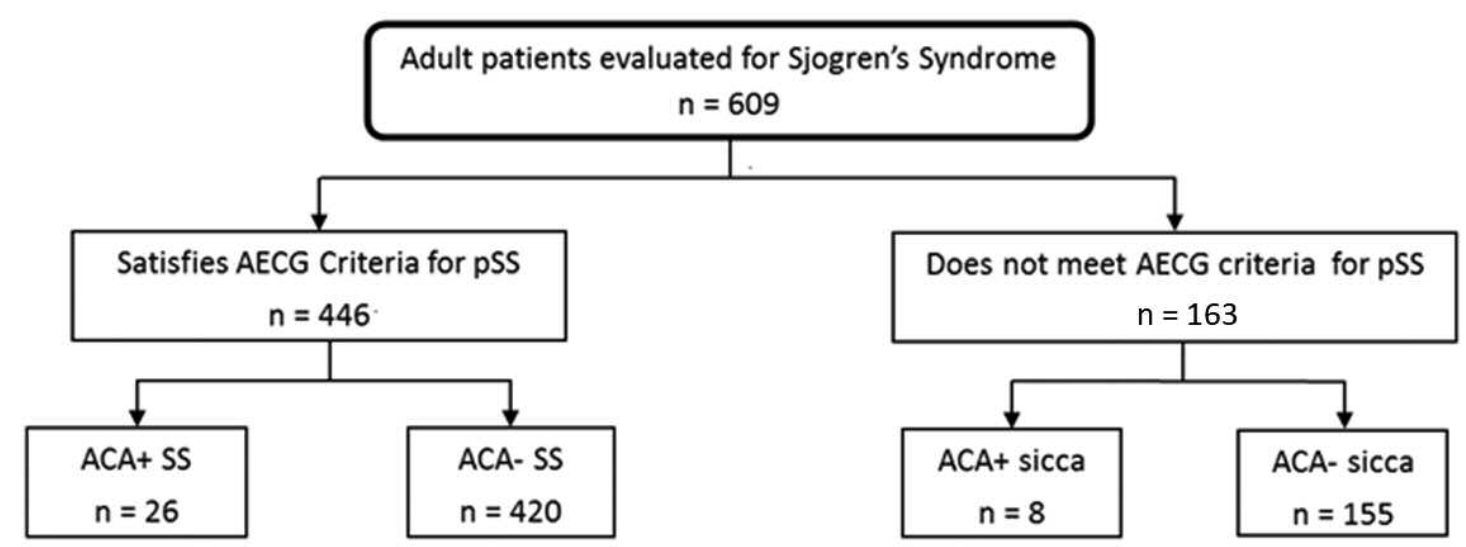

Figure 1. Flowchart for patient enrollment. AECG: American-European Consensus Group; SS: Sjögren syndrome; pSS: primary SS; ACA: anticentromere antibody.

SSc-SS overlap also displayed a classic SS phenotype with recurrent salivary gland enlargement, purpura, fatigue, arthralgia, and leukocytopenia ${ }^{7,8}$.

ACA is positive mainly in patients with SSc who have CREST features ${ }^{15}$ and has been detected in $50-96 \%$ of CREST syndrome patients ${ }^{9}$. The prevalence of ACA in SS has been reported as $1.4-10.85 \%, 7,8,9,10$. In our cohort, positive ACA was seen in $5.8 \%$ of our patients with pSS. We have demonstrated that among pSS patients who are also $\mathrm{ACA}+$, there is more severe dryness of the eyes as measured by the S1T, subjective xerostomia on VAS, and objective loss in saliva production (USSF). More patients with ACA+ SS had a focus score $>1$ than did patients with ACA-pSS.

Previous studies have shown that patients with ACA+ SS 
have a lower prevalence of anti-Ro or anti-La antibodies ${ }^{16,17,18}$ compared with $\mathrm{ACA}-\mathrm{SS}^{19,20}$. Among patients with SSc-SS, there is a $21-33 \%$ prevalence of positive anti-Ro antibodies ${ }^{2,16,18}$. Other groups have found that about $60 \%$ of their SSc patients with sicca symptoms were positive for anti-Ro antibodies ${ }^{2,17}$. Our results show a similar prevalence of anti-Ro or anti-La antibodies in $35 \%$ of patients with ACA+SS.

The lower prevalence of anti-Ro and anti-La antibodies in the ACA+ SS group indicates that many of these patients depended upon the finding of a positive MSG biopsy to meet the AECG classification criteria. These are overlap cases and many would have been missed if classification as pSS depended upon the finding of anti-Ro/La antibodies (i.e., it is necessary to do the MSG biopsy to diagnose many ACA+ patients as having SS).

Avouac, et al notes that in biopsy samples from 50 (55\%) of 91 patients with SSc and sicca complaints, they observed fibrotic lesions (considered mild in 13, moderate in 17, and severe in 20), and samples from 18 of the 91 patients $(20 \%)$ had a focus score $\geq 1^{2}$. Even though there is more fibrotic MSG involvement in patients with SSc with sicca ${ }^{2}$, patients with ACA+ SS are dry because of true inflammation in the salivary glands. Our results did not show a significant difference in MSG fibrosis between patients with ACA+ SS and patients with ACA-SS. We found a significantly higher proportion of patients with ACA+ SS with focus score $\geq 1$ (92\% vs 84\%). However, there did not appear to be a difference between the focus scores of the patients with $\mathrm{ACA}+\mathrm{SS}$ and those with ACA-SS.

This raises major questions about the essential difference between progressive SSc and ACA+ lcSSc, where the overlap with SS is more frequent than would be expected, and the MSG biopsy pathology is more like SS than like SSc.

Sicca syndrome is common in SSc (60\%) and is associated with salivary fibrosis ${ }^{7}$, more severe disease, and a higher mortality rate $^{2}$. Abnormalities of collagen gene transcription may be responsible for tissue and vascular fibrosis, causing glandular fibrosis in $\mathrm{SSc}^{2,21}$. The pathology in the patients with ACA+ SS does not seem to follow this morphology.

A limitation of comparing the patients with $\mathrm{ACA}+\mathrm{SS}$ and those with ACA-SS in our study is that there are relatively few patients with ACA+ SS. Thus, each patient with ACA+ $\mathrm{SS}$ represents a greater fraction of the overall ACA+ SS group. However, our results show that there is a highly significant difference in severity of sicca symptoms and objective severity between the 2 groups.

Our findings indicate that the presence of ACA is associated with more severe xerostomia symptoms in patients diagnosed with pSS. ACA + SS is associated with objective measures of more profound xerostomia and xerophthalmia compared to ACA-SS. Further, there is a higher prevalence of RP and a lower prevalence of anti-Ro and anti-La serology in patients with ACA+ SS. Aside from a difference in RP prevalence, there were no statistically significant differences in extraglandular manifestations between the 2 groups.

Last, the majority of patients with ACA+ SS meet the AECG criteria for $\mathrm{pSS}$ despite having negative serology for anti-Ro or anti-La antibodies. This may suggest that anti-Ro or anti-La serology may be less useful when trying to diagnose pSS in ACA+ patients. In view of evolving potential therapeutic tools for SS, and the finding of inflammatory rather than fibrotic pathology in ACA+ SS, our study also highlights the importance of doing an MSG biopsy in ACA+ patients with objective evidence of decreased salivary flow, decreased S1T, or abnormal van Bijsterveld staining. We take note of the essential difference in pathology of the MSG biopsy in ACA+ patients with sicca complaints compared to progressive SSc patients with similar sicca symptoms.

\section{REFERENCES}

1. Hollander JL. Arthritis and allied conditions: a textbook of rheumatology. Philadelphia: Lea and Febiger; 1960:988.

2. Avouac J, Sordet C, Depinay C, Ardizonne M, Vacher-Lavenu MC, Sibilia J, et al. Systemic sclerosis-associated Sjögren's syndrome and relationship to the limited cutaneous subtype: results of a prospective study of sicca syndrome in 133 consecutive patients. Arthritis Rheum 2006;54:2243-9.

3. Vitali C, Bombardieri S, Jonsson R, Moutsopoulos HM, Alexander EL, Carsons SE, et al; European Study Group on Classification Criteria for Sjögren's Syndrome. Classification criteria for Sjögren's syndrome: a revised version of the European criteria proposed by the American-European Consensus Group. Ann Rheum Dis 2002;61:554-8.

4. Shiboski SC, Shiboski CH, Criswell L, Baer A, Challacombe S, Lanfranchi H, et al; Sjögren's International Collaborative Clinical Alliance (SICCA) Research Groups. American College of Rheumatology classification criteria for Sjögren's syndrome: a data-driven, expert consensus approach in the Sjögren's International Collaborative Clinical Alliance cohort. Arthritis Care Res 2012;64:475-87.

5. Bongi SM, Melchiorre D. Primary Sjögren's syndrome: diagnostic criteria, diagnostic aspects and controversies. Pain Clin 2002;13:293-304.

6. Baldini C, Giusti L, Bazzichi L, Lucacchini A, Bombardieri S. Proteomic analysis of the saliva: a clue for understanding primary from secondary Sjögren's syndrome? Autoimmun Rev 2008; 7:185-91.

7. Salliot C, Mouthon L, Ardizzone M, Sibilia J, Guillevin L, Gottenberg JE, et al. Sjögren's syndrome is associated with and not secondary to systemic sclerosis. Rheumatology 2007;46:321-6.

8. Baldini C, Mosca M, Della Rossa A, Pepe P, Notarstefano C, Ferro F, et al. Overlap of ACA-positive systemic sclerosis and Sjögren's syndrome: a distinct clinical entity with mild organ involvement but at high risk of lymphoma. Clin Exp Rheumatol 2013;31:272-80.

9. Nakamura H, Kawakami A, Hayashi T, Iwamoto N, Okada A, Tamai M, et al. Anti-centromere antibody-seropositive Sjögren's syndrome differs from conventional subgroup in clinical and pathological study. BMC Musculoskelet Disord 2010;11:140.

10. Hayashi N, Koshiba M, Nishimura K, Sugiyama D, Nakamura T, Morinobu S, et al. Prevalence of disease-specific antinuclear antibodies in general population: estimates from annual physical examinations of residents of a small town over a 5-year period. Mod Rheumatol 2008;18:153-60.

Personal non-commercial use only. The Journal of Rheumatology Copyright @ 2020 . All rights reserved. 
11. Kassan SS, Moutsopoulos HM. Clinical manifestations and early diagnosis of Sjögren syndrome. Arch Gen Intern Med 2004; 164:1275-84.

12. Prause JU, Kriegbaum NJ, Manthorpe R, Oxholm P. Rose bengal score - a possible key parameter when evaluating disease level and progression in primary Sjögren's syndrome. J Autoimmun 1989;2:501-7.

13. Knezovic I, Alajbeg I, Karlovic D, Pavan J, Vrkic N, Biscan A. Differential diagnostic performance of Rose Bengal Score Test in Sjögren's syndrome patients. Coll Antropol 2011;35:1105-13.

14. Speight PM, Kaul A, Melsom RD. Measurement of whole unstimulated salivary flow in the diagnosis of Sjögren's syndrome. Ann Rheum Dis 1992;51:499-502.

15. Miyawaki S, Asanuma H, Nishiyama S, Yoshinaga Y. Clinical and serological heterogeneity in patients with anticentromere antibodies. J Rheumatol 2005;32:1488-94.

16. Osial TA Jr., Whiteside TL, Buckingham RB, Singh G, Barnes EL, Pierce JM, et al. Clinical and serologic study of Sjögren's syndrome in patients with progressive systemic sclerosis. Arthritis Rheum 1983;26:500-8.
17. Bell S, Krieg T, Meurer M. Antibodies to Ro/SSA detected by ELISA: correlation with clinical features in systemic scleroderma. Br J Dermatol 1989;121:35-41.

18. Drosos AA, Andonopoulos AP, Costopoulos JS, Stavropoulos ED, Papadimitriou CS, Moutsopoulos HM. Sjögren's syndrome in progressive systemic sclerosis. J Rheumatol 1988;15:965-8.

19. Katano K, Kawano M, Koni I, Sugai S, Muro Y. Clinical and laboratory features of anticentromere antibody positive primary Sjögren's syndrome. J Rheumatol 2001;28:2238-44.

20. Hida A, Kawabe Y, Kawakami A, Migita K, Tominaga M, Nakamura H, et al. HTLV-I associated Sjögren's syndrome is aetiologically distinct from anti-centromere antibodies positive Sjögren's syndrome. Ann Rheum Dis 1999;58:320-2.

21. Jimenez SA, Derk CT. Following the molecular pathways toward an understanding of the pathogenesis of systemic sclerosis. Ann Gen Intern Med 2004;140:37-50. 